\title{
Differences in Population Dynamics and Uptake of Reproductive Health Services in the Urban and Rural Cohorts of Cross River Health and Demographic Surveillance System of Southern Nigeria
}

\author{
Iwara Arikpo $^{1,2, *} \quad$ Anthony Okoro ${ }^{1,2}$ Ekpereonne Esu ${ }^{1,3} \quad$ Ememobong Aquaisua $^{1}$ \\ Ideba Mboto ${ }^{1,2}$ Martin Meremikwu ${ }^{1,4}$ \\ 1. Cross River Health \& Demographic Surveillance System, University of Calabar \\ 2. Department of Computer Science, University of Calabar \\ 3. Department of Public Health, University of Calabar \\ 4. Department of Paediatrics University of Calabar
}

\begin{abstract}
Background: Health and demographic surveillance systems (HDSSs) generate essential health and demographic data from longitudinal surveillance of populations resident in distinctly demarcated enumeration areas. The Cross River HDSS (CRHDSS) located in southern Nigeria includes distinct urban and rural cohorts. Concurrent surveillance of adjacent urban and rural communities provides an opportunity to identify differences in population characteristics and access or utilization of health services. In this paper, we report the result of comparative analysis of rural and urban cohorts of the CRHDSS from November 2012 to December 2018. Methodology: Data was collected through house-to-house interviews performed in 6-monthly cycles through the years. Information obtained included demographic characteristics, pregnancies, child birth, health-seeking behaviour, migration and deaths. Trained field workers collected data from all households in the demographic surveillance area with mobile Android devices (running the ODKCollect). Collected data were transferred electronically to a remote server running on ODKAggregate and further processed by MirthConnect on OpenHDS web application with a MySQL backend database. Data was exported, cleaned and analyzed with the R Statistical Computing Software. Results: Total population undergoing longitudinal health and demographic surveillance was 37,808 persons in 9,452 households with 18,414 males and 19,394 females. The rural cohort made up $47.4 \%$ of the population while the urban cohort was $52.6 \%$. Population structure showed that while the rural cohort has a relative depletion of young adults, the urban cohort has preponderance of this population segment indicating the high rate of rural to urban migration. There is also a striking difference in pattern of utilization of reproductive health services by pregnant women. While $68.6 \%$ of childbirth in the rural cohort occurred outside the formal health service (at TBAs and at home), $79.1 \%$ of childbirth in the urban area occurred in formal health facilities. Both the crude fertility rate, infant mortality and under-five mortality rates were higher in rural than the urban cohorts.Conclusion: Surveillance data showed relative depletion of young adults from rural population cohort, suggesting high rate of rural to urban migration. Majority of childbirths in rural areas still occur outside formal health facilities, which may partly contribute to higher infant mortality rate in the rural cohort. This calls for more efforts to enhance reproductive health services and employment opportunities in rural areas.
\end{abstract}

Keywords: Population, Health characteristics, births, deaths, health and demographic surveillance systems DOI: $10.7176 / \mathrm{DCS} / 9-5-08$

Publication date:May $31^{\text {st }} 2019$

\section{Introduction}

Data on socioeconomic characteristics of households, health status and demographic changes of populations are vital in monitoring trends and for economic planning and projections. Governments, researchers and policy makers need population and health data to determine qualities and characterization of essential social, health and economic factors such as age distribution, sex distribution, population density, population dynamics, health status and vital statistics among others. As far back as 1866 , census exercise, vital registration and surveys have been the major sources of these data collection in Nigeria (National Population Commission, 2014). The outcomes are used to estimate the economic growth of a nation amidst the drawbacks of these data collection approaches.

Developing countries are faced with difficulty in collecting these demographic data correctly, due to decennial censuses (often last more than 10 years in most cases), poor health management systems and irregular household surveys (Bos, 2004). For instance, there are several factors that affect the disparities in the results of census data between the urban and rural communities. Low response rates are often experienced in rural areas where there is higher number of people with lower education level and lower literacy abilities compared to the urban dwellers. In most cases, the poor terrain of the rural residents also contributes to the disproportionate census figures. This is because many homes are located in remote and hard-to-reach areas (Ikande, 2018). 
The near absence of good working civil registration and vital statistics system (CRVS) in low- and mediumincome countries (LMICs), such as Nigeria, also limits the effort of economic planners to make effective decisions on policies (Ye, Wamukoya, Ezeh, Emina, \& Sankoh, 2012). The overall impact falls on the inability of economic planners to make distributive projections and forecasts on population growth in rural and urban settlements. Again, lifestyle patterns/behaviours, access to social amenities, cultural beliefs, and urbanization affect the rate of population growth of a society.

In a study done by Ottong, Ering, \& Akpan (2010), it was shown that though the population of Cross River State experienced a rapid growth between 1991 and 2006, there was relatively low skilled manpower to withstand the prevailing demographic features. Data such as this is essential in helping government to make projections and invest more in education as one way of sustaining the economic growth. Proper tracking of the population situation is therefore vital for the socioeconomic development of the society.

Health and demographic surveillance system (HDSS) sites help to improve the national vital registration system, and also address the challenges inherent in the CRVS procedures by creating a platform that enables researchers monitor population over time and make comparative analysis on the demographic changes, household characteristics like birth practices, deaths, migrations, education, occupation and work status in their demographic surveillance areas (DSAs). HDSS have proven to be a reliable means of generating longitudinal data from distinctly demarcated enumeration areas to guide countries (in settings where the vital registration systems or health management information systems are weak) in setting up priorities on health and demographic changes based on evidences from routine longitudinal household data collection (Kwei, 2006). Some of the indicators tracked by HDSS sites include: migrations, births, deaths, causes of deaths, pregnancies, as well as socioeconomic characteristics.

The Cross River Health and Demographic Surveillance System (Cross River HDSS) located in Southern Nigeria is one of such research platforms established in 2010 to, among other objectives, generate timely and reliable information necessary to inform policy makers on differences in population characteristics (Arikpo, et al., 2013) and access or utilization of health services. The routine house-to-house update cycles ensure that all households in the Enumeration Areas (EAs) are visited at every round and all vital events are tracked. This makes it possible for new entrants into the Demographic Surveillance Area (DSA) to be captured and all other vital events tracked.

This paper focuses on the comparative analysis of demographic and socioeconomic characteristics and the uptake of reproductive health services in the rural and urban cohorts of the Cross River HDSS from November 2012 to December 2018. It describes the procedures of HDSS operations and further presents a comparative analysis of the characteristics of both cohorts. The research methodology, which describes the study area and data collection procedures, is presented in the next section. Subsequent sections present the results and discussion, as well as the conclusion of this paper.

\section{Methodology}

\subsection{Study Area}

The Cross River health and demographic surveillance system (Cross River HDSS) operates two research cohorts located within the southern senatorial district of Cross River State in south-south Nigeria, with a combined population of 37,808 persons in 9,452 households as at 2018 (about $47.4 \%$ of which are rural dwellers). The first is a rural cohort located in the Akpabuyo Local Government Area (LGA) of the state and the second, an urban cohort located in Calabar Municipal, the state capital (Figure 1). The rural and urban sites are further delineated into 46 and 43 contiguous Enumeration Areas (EAs) respectively. Calabar Municipal, with an area of 142.74 $\mathrm{km}^{2}$, is located on $4^{\circ} 58^{\prime} 28.056^{\prime \prime}$ North and longitude $8^{\circ} 20^{\prime} 29.9328^{\prime \prime}$ East. The city majorly comprises of the Quas and Efiks ethnic groups. Similarly, Akpabuyo LGA, spanning an area of $813.68 \mathrm{~km}^{2}$, (National Population Commission, 2010) is located on $4^{\circ} 57^{\prime} 0.4248^{\prime \prime}$ North and longitude $8^{\circ} 23^{\prime} 35.9088^{\prime \prime}$ East. In addition to the Quas and Efiks found in Calabar Municipal, Akpabuyo LGA also has the Efuts ethnic group. Both sites are situated in the tropical rain forest belt of southern Nigeria, with an annual rainfall in the range of $2500 \mathrm{~mm}$ to $3000 \mathrm{~mm}$ and mean annual temperature of $30^{\circ} \mathrm{C}$. 


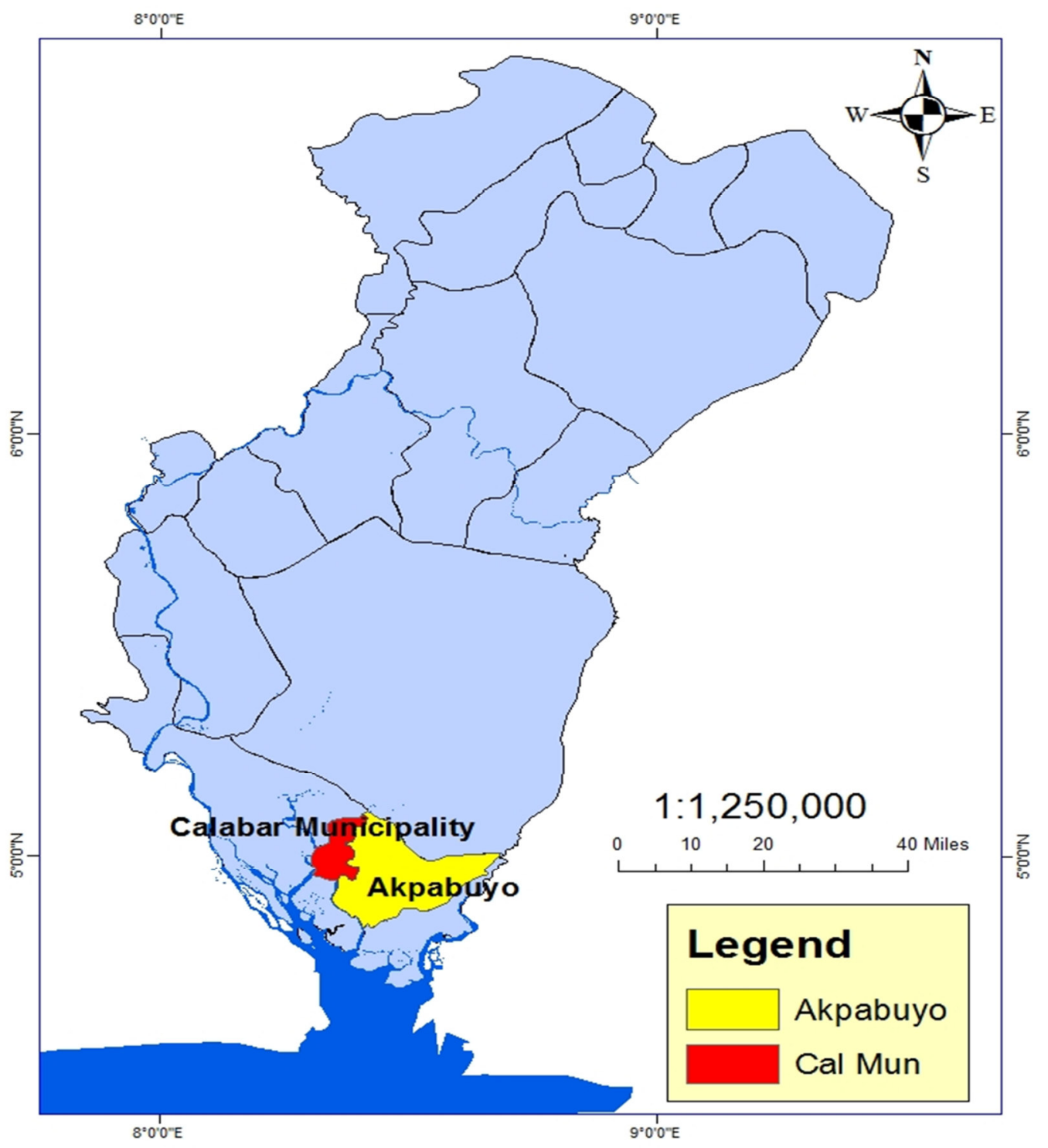

Figure 1. Map showing urban and rural cohorts of Cross River HDSS

\subsection{Data Procedures}

The INDEPTH Network (International Network of Field Sites with continuous Demographic Evaluation of Populations and Their Health) has many tested and standardized forms (questionnaires) for the core HDSS processes which are used for routine data collection in all 42 Research Centres with 49 HDSS Field Sites across Africa, Asia and the Oceania, including the Cross River HDSS (INDEPTH Network, 2019). The specific forms used to generate the data for this paper are, Household Registration Book (HRB), Household \& socioeconomic characteristics, Pregnancy observation and outcome, and Death registration, respectively. At the inception of our full site (urban \& rural cohorts) in November 2012 (after a pilot), routine data collection was performed in 4monthly cycles per year. This was however, changed in 2016 with a switch to a 6-monthly cycle per year. Each of these cycles is called an update round in HDSS parlance.

At every update round visit, trained field workers administer the respective questionnaires to all households in the demographic surveillance area (DSA) to collect information on vital events such as pregnancies, births, deaths and migrations. Data on the demographic characteristics and health-seeking behavior of these visited households are also collected. This data collection is done with mobile devices running the Android operating system. These Android devices run the ODKCollect (Open Data Kit, 2019) to capture events, and Mobile interop server for viewing of event data before transmitting using MirthConnect middleware application to MySQL database running behind the OpenHDS, an open source software for managing longitudinal data for health and demographic surveillance systems (SwissTPH, 2019). Data was extracted from the OpenHDS, and analyzed using a free and open source software, the R Statistical Computing Software (The R Foundation, 2019). 


\section{Results}

Population pyramids

CRHDSS Akpabuyo (rural) Site pyramid as @ 2018

$$
\text { Pop. }=17936
$$
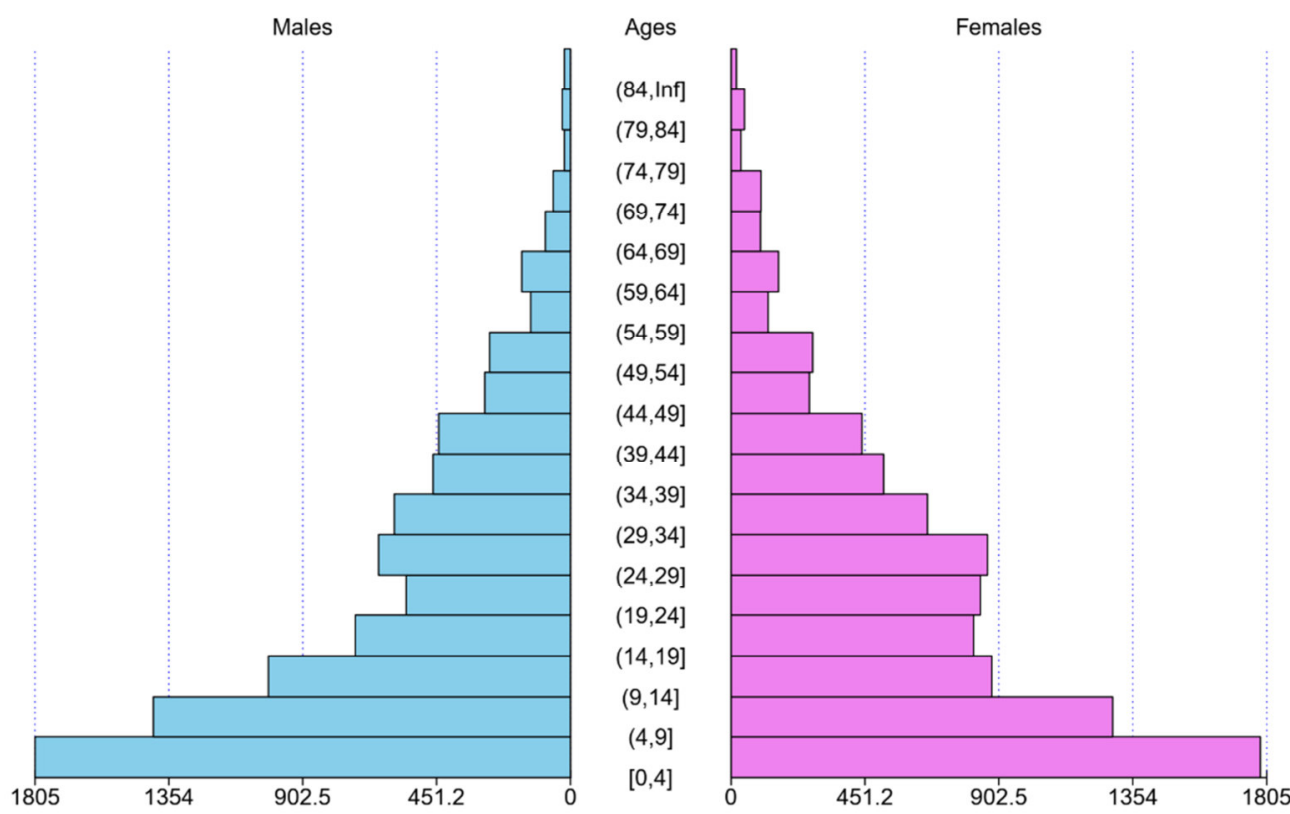

Figure 2. Population pyramid for Akpabuyo

CRHDSS Calabar-Municipal Site pyramid as @ 2018 Pop. = 19867
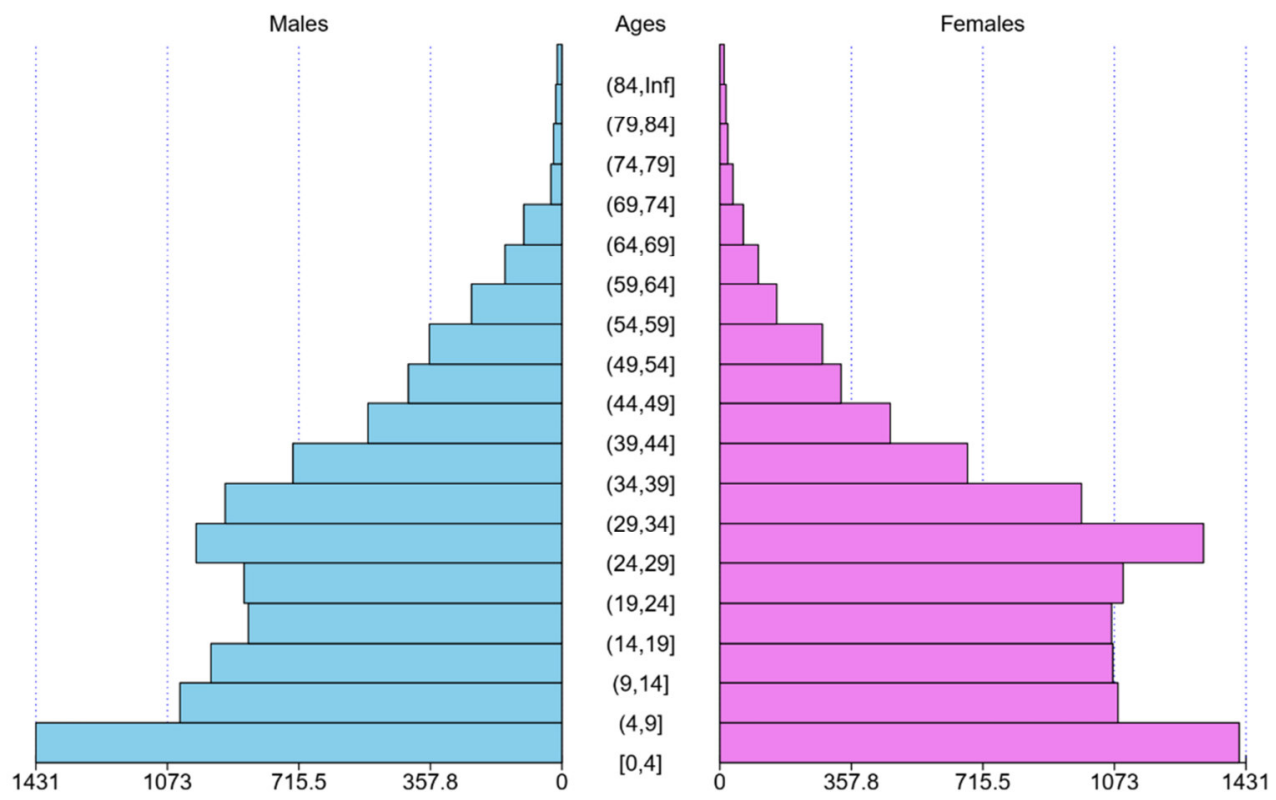

Figure 3. Population pyramid for Calabar Municipal 

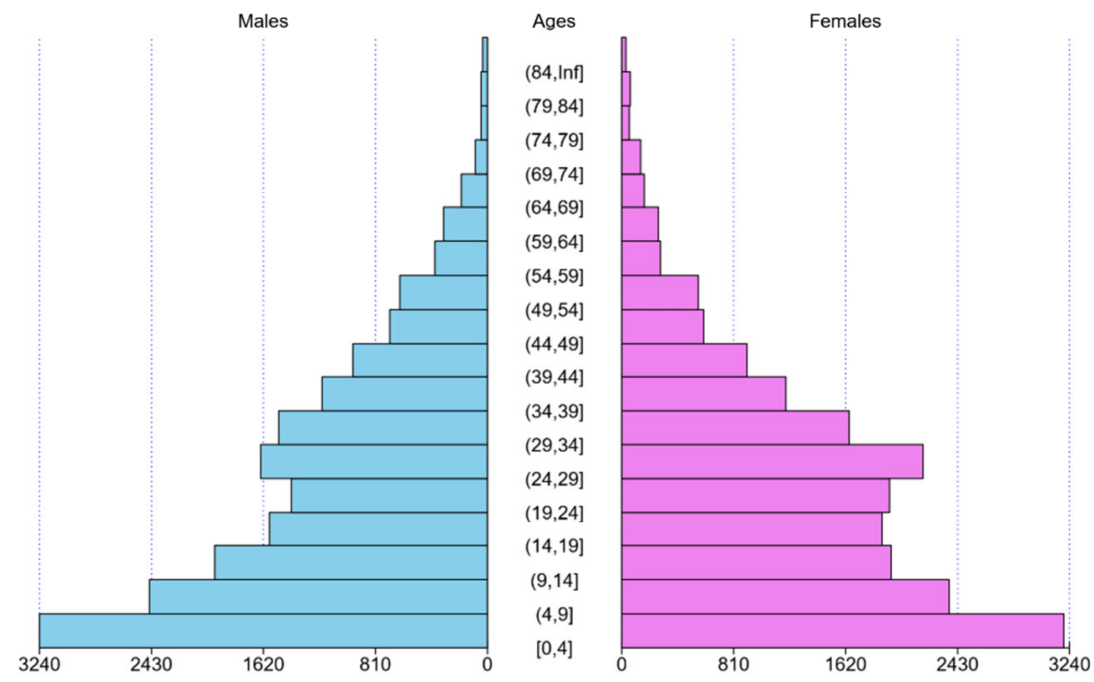

Figure 4. Population pyramid for Akpabuyo \& Calabar Municipal

Table 1. Vital statistics \& fertility as at 2018

\begin{tabular}{lrrrr}
\hline Indicator/Rate & Rural Only & Urban Only & \multicolumn{2}{c}{ Rural \& Urban } \\
\hline Total Resident Population & 17606 & 19619 & 37228 \\
No. of Households & 4638 & 4814 & 9452 \\
No. of pregnancies observed & 332 & 212 & 544 \\
No. of Live births & 994 & 652 & 1646 \\
Crude Birth Rate (CBR) & 56 per 1000 person-years & 33 per 1000 person-years & 44 per 1000 person-years \\
Crude Death Rate (CDR) & 18 per 1000 person-years & 7 per 1000 person-years & 12 per 1000 person-years \\
\hline
\end{tabular}

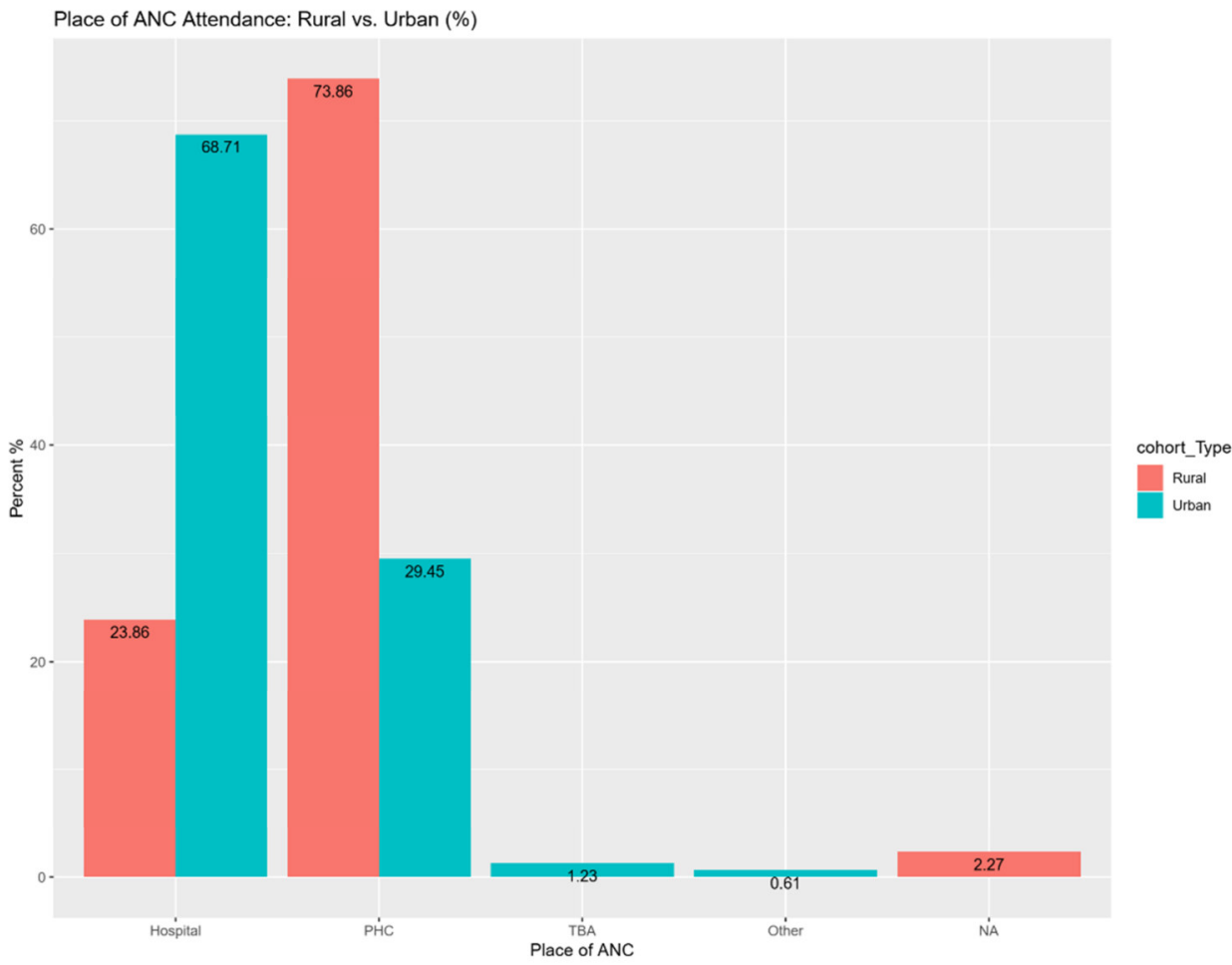

Figure 5. Place of ANC attendance in urban and rural cohorts 


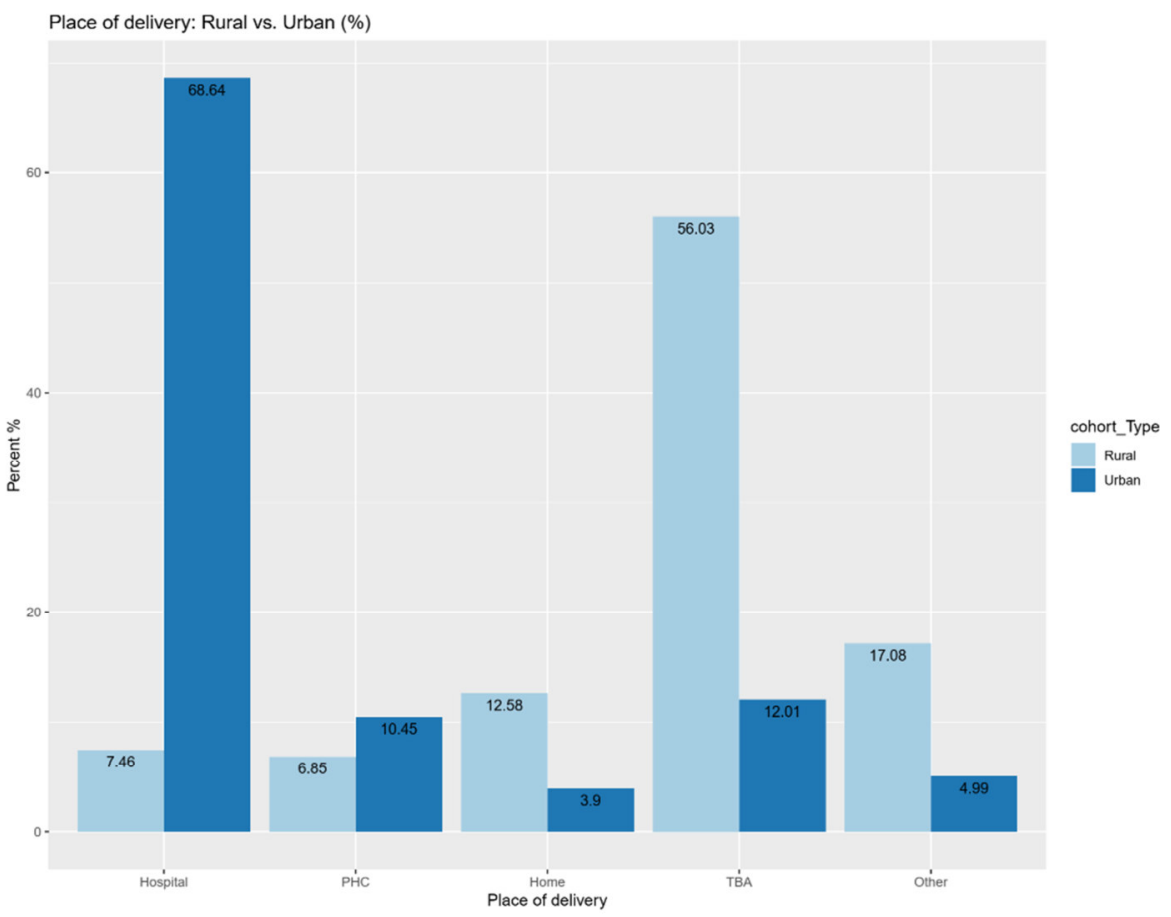

Figure 6. Place of delivery in urban and rural cohorts

Table 2. Mortality rates as at 2018

\begin{tabular}{lrrr}
\hline Indicator/Rate & \multicolumn{1}{c}{ Rural Only } & Urban Only & \multicolumn{1}{c}{ Rural \& Urban } \\
\hline Crude Death Rate (CDR) & 18 per 1000 person-years & 7 per 1000 person-years & 12 per 1000 person-years \\
Neonatal mortality & 4 per 1000 births & - & 2 per 1000 births \\
Infant mortality & 16 per 1000 births & - & 9 per 1000 births \\
Under-5 mortality & 50 per 1000 births & 6 per 1000 births & 32 per 1000 births \\
\hline
\end{tabular}

\section{Discussion}

From the population pyramids in Figures 2, 3, and 4, more than 48\% of the male population are aged 0-14 years and about $43 \%$ of the female population are aged 0-14 years in the Akpabuyo (rural) cohort; while in the Calabar Municipal (urban) cohort, the population of males $0-14$ years is $35.4 \%$ and that of females of $0-14$ years is $35 \%$. The dependent population (i.e., $0-14$ and $65+$ years) make up $48.4 \%$ of the entire population in the Akpabuyo cohort, and $36.9 \%$ in the Calabar Municipal cohort. These values show that there is higher dependency burden on the productive class (15-64 years) in the rural cohort compared to that of urban. It implies that the economically active population would face more burden daily especially in the rural settlements where literacy levels and employment are generally lower than in the urban areas. The overall economy is also affected because the households will likely consume more than what is produced. Though there are debates that not all persons above the age of 65 are economically dependent, likewise not all persons aged 15 to 65 years are economically active (Holdsworth, Gould, Finney, Marshall, \& Norman, 2013).

Similarly, this surveillance data showed that percentage of the population aged $19-38$ years is $28.6 \%$ in the rural cohort compared to $38.1 \%$ in the urban cohort. This relative depletion of young adults from rural population cohort suggests high rate of rural to urban migration; thereby contributing to more burden on few dependent individuals present in the households. This appears to also contribute significantly to the higher death rates of the dependent population seen in the rural cohort as compared with that of the urban. As depicted in Table 2, there is a huge gap between the proportions of deaths in the rural cohort compared to the urban. For instance, the crude death rate (CDR) in the rural cohort is more than twice the value for the urban cohort. Interventions on education, social security and healthcare services are possible solutions, and should be strengthened to reduce the burden on the economically-active population. In addition, establishment of loans and skills acquisition centres, creation of industries such as Agro-based cottage industries in the rural areas are possible avenues to reduce the rural-urban migration of young adults who migrate to the city in search of better means of livelihood.

Another variation in this comparative analysis is on the level of utilization of reproductive health services (particularly place of child birth) in the study population. The provision of free medical services to pregnant women and children under five years by Cross River State Government notwithstanding, childbirth practices in 
the rural settings of the state predominantly take place outside the formal health facilities. As shown in Figure 6 , a whopping $85.7 \%$ of pregnant women delivered outside the formal health facilities, with only about $14.3 \%$ of deliveries occurring in formal health facilities. This is in clear contrast with results from the urban where 79.1\% of births occurred in formal health facilities. This underpins the need for stakeholders to work to discourage rural women from seeking care outside the formal health facilities. Studies have shown that proximity and accessibility to TBAs' (traditional birth attendance) homes, personal relationships between the TBA and the pregnant woman or other relations, affordable charges, religious affiliations, cultural beliefs, fear of injections and tablets, availability of 'special herbs' perceived to be effective for pregnancy and child birth, amongst others have over time greatly influenced the preference of TBAs (Akpabio et al., 2014; Okafor et al., 2014; Adegoke \& Jegede, 2016). Nevertheless, it is quite commendable to see the high rate of ANC attendance across the cohorts (Figure 5). The study showed that the urban cohort had higher ANC attendance in the Hospital (68.7\%) as against rural (23.9\%). For the ANC attendance in PHCs, the percentage attendance in the rural cohort was higher $(73.9 \%)$ while urban recorded $23.5 \%$ attendance. This could be attributed to the availability and accessibility of these categories of health facilities in both cohorts.

Adoption of standard and recommended family planning methods appears to be less practiced in the rural cohort compared to the urban cohort. For instance, the number of women of reproductive age (15-49 years) in the rural cohort constitute $24.5 \%$ and $29.9 \%$ in the urban cohort. A combination of both cohorts puts women of reproductive age at $27.3 \%$. This is evident in the broader base of the population pyramid of the rural cohort than that of the urban cohort at this age group/level. Despite the higher number of women in reproductive age in the urban cohort, households in the rural cohort still had higher number of pregnancies (Table 1) and higher number of under-five children (20\% in the rural cohort and about $14.3 \%$ in the urban cohort). Figures $2-4$, show the population pyramids for Cross River HDSS cohorts as at 2018. The crude birth rate (CBR) in the rural is also higher compared to the value in the urban cohort. An obvious evidence points to the fact that, there are more births, pregnancies and deaths, especially infant mortalities in the rural cohort compared to the urban. This calls for more intervention of government and NGOs in health care to further increase the awareness on reproductive health in these areas. Effort should be intensified in ensuring the functioning of family planning clinics in every health facility.

Effective tracking of pregnancies, births amongst other socio-demographic events are reliable strategy to gather reliable information that can inform policy and improve quality and access to care. The success of implementing this strategy on a 6-monthly cycle do however have limitations which include increased likelihood of missing some pregnancies and birth outcomes especially stillbirths. This calls for the strengthening of Community Key Informants (CKIs) system in the field operations of HDSS sites.

\section{Conclusion}

This paper reported the differences in population dynamics and uptake of health services in the rural and urban cohorts of the Cross River HDSS. These cohorts represent typical rural and urban settings in southern Nigeria, hence, the results presented in this paper can serve as a useful guide to stakeholders seeking to understand, and act on rural-urban variations, as well as the impact of the reproductive health care available in such climes. Because of the peculiar intensive community-based nature of longitudinal studies like health and demographic surveillance systems, the results from this study may slightly vary with survey-related results. However, the former serves to fill gaps in survey-related studies, a reason, countries like Ghana put high premium on the data from the three health and demographic surveillance systems in that country. This is an increasing practice in countries where health and demographic surveillance systems are running. The ability to continuously track most events in communities under surveillance over time further highlights health and demographic surveillance systems as reliable research platforms capable of strengthening the vital registration as well as health information systems.

\section{References}

Adegoke, O., \& Jegede, A. (2016). Continued Patronage of Traditional Birth Attendants (TBAs) by Pregnant Women in a Traditional African Community. Annals of Public Health and Research, 3(3).

Akpabio, I., Edet, O., Etifit, R., \& Robinson-Bassey, G. (2014). Women's preference for traditional birth attendants and modern health care practitioners in Akpabuyo community of Cross River State, Nigeria. Health Care Women Int, 35(1), 100-109. doi:10.1080/07399332.2013.815751

Arikpo, I., Mboto, I., Okoro, A., James, U., Aquaisua, E., Osonwa, K., . . . Meremikwu, M. (2013). Optimum hardware, software and personnel requirements for a paperless health and demographic surveillance system: a case study of Cross River HDSS, Nigeria. Information and Knowledge Management, 3(3), 77-83.

Bos, E. (2004). Population and Health in Developing Countries. International Journal of Epidemiology, 33(4), 916-917. doi:https://doi.org/10.1093/ije/dyh220

Herbst, K., Juvekar, S., Bhattacharjee, T., Bangha, M., Patharia, N., Tei, T., . . . Sankoh, O. (2015). The 
INDEPTH Data Repository: An International Resource for Longitudinal Population and Health Data From Health and Demographic Surveillance Systems. Journal of empirical research on human research ethics, 10(3), 324-333. doi:10.1177/1556264615594600

Holdsworth, C., Gould, W., Finney, N., Marshall, A., \& Norman, P. (2013). Population and Society. London: SAGE Publications Ltd.

Ikande, M. (2018). Problems of population census in Nigeria. Retrieved from The Leadership Website: https://www.legit.ng/1133808-problems-population-census-nigeria.html

INDEPTH Network. (2019). INDEPTH Network. Retrieved April 17, 2017, from INDEPTH Network: http://www.indepth-network.org/

Kwei, R. (2006). Profiles, INDEPTH Network Leading research in Africa and Asia. Retrieved from Eyes on Malaria Online: http:/www.eyesonmalaria.org/content/profiles-indepth-network-leading-research-africaand-asia

National Population Commission. (2010). Census 2006 Priority Tables Volum III. Abuja, Nigeria: National Population Commission.

National Population Commission. (2014). Nigeria Demographic and Health Survey. Abuja, Nigeria: ICF International.

Okafor, I., Sekoni, A., Ezeiru, S., Ugboaja, J., \& Inem, V. (2014). Orthodox versus unorthodox care: A qualitative study on where rural women seek healthcare during pregnancy and childbirth in Southwest, Nigeria. Malawi Medical Journal, 26(2), 45-49.

Open Data Kit. (2019). Retrieved July 01, 2016, from OpenDataKit: https://opendatakit.org/

SwissTPH. (2019). GitHub - SwissTPH/openhds. Retrieved July 2016, from SwissTPH/openhds: https://github.com/SwissTPH/openhds

The R Foundation. (2019). Retrieved June 2016, from The R Project for Statistical Computing: https://www.rproject.org/

United Nations. (n.d.). Dependency Ratio. Retrieved from United Nations: http://www.un.org/esa/sustdev/natlinfo/indicators/methodology_sheets/demographics/dependency_ratio.pdf

Ye, Y., Wamukoya, M., Ezeh, A., Emina, J., \& Sankoh, O. (2012). Health and demographic surveillance systems: a step towards full civil registration and vital statistics system in sub-Sahara Africa? BMC Public Health, 12(741). doi:10.1186/1471-2458-12-741 